LAWRENCE LIVERMORE N A T IO N A L LABORATORY

\section{A Comparison of Detailed Level and Superconfiguration Models of Neon}

S. B. Hansen , K. B. Fournier, C. Bauche-Arnoult, J. Bauche, O. Peyrusse

January 19, 2005

Radiative Properties of Hot Dense Matter Santa Barbara, CA, United States

November 1, 2004 through November 5, 2004 
This document was prepared as an account of work sponsored by an agency of the United States Government. Neither the United States Government nor the University of California nor any of their employees, makes any warranty, express or implied, or assumes any legal liability or responsibility for the accuracy, completeness, or usefulness of any information, apparatus, product, or process disclosed, or represents that its use would not infringe privately owned rights. Reference herein to any specific commercial product, process, or service by trade name, trademark, manufacturer, or otherwise, does not necessarily constitute or imply its endorsement, recommendation, or favoring by the United States Government or the University of California. The views and opinions of authors expressed herein do not necessarily state or reflect those of the United States Government or the University of California, and shall not be used for advertising or product endorsement purposes. 


\title{
A Comparison of Detailed Level and Superconfiguration Models of Neon
}

\author{
S.B. Hansen ${ }^{\mathrm{a}, *}$ K.B. Fournier ${ }^{\mathrm{a}}$ C. Bauche-Arnoult ${ }^{\mathrm{b}}$ J. Bauche ${ }^{\mathrm{b}}$ \\ O. Peyrusse ${ }^{\mathrm{c}}$ \\ ${ }^{a}$ Lawrence Livermore National Laboratory, P.O. Box 808, L-473,Livermore, CA \\ 94550, USA \\ ${ }^{\mathrm{b}}$ Laboratoire Aimé Cotton, Campus d'Orsay, Bâtiment 505, 91405 Orsay, France \\ ${ }^{\mathrm{c}}$ CELIA, UMR 5107 Université Bordeaux I - CEA - CNRS, 33405 Talence, France
}

\begin{abstract}
The superconfiguration (SC) approach to collisional-radiative modeling can significantly decrease the computational demands of finding non-LTE level populations in complex systems. However, it has not yet been fully determined whether the statistical averaging of SC models leads to a significant loss of accuracy. The present work compares results from two independent models: a detailed-level accounting (DLA) model based on HULLAC data and the SC model MOST. The relatively simple level structures of the K- and L-shell ions of the neon test system ensure a tractable number of levels in the DLA model but challenge the statistical assumptions of the SC approach. Nonetheless, we find fair agreement between the two models for average ion charges, SC populations, and various effective temperatures.
\end{abstract}

Key words: Superconfiguration, Collisional-radiative, Non-LTE, Effective temperature

\section{Introduction}

Effective temperatures governing the population distributions of energy levels in plasma ions have long held promise as a way to ease the significant computational burden of non-LTE modeling [1-3]. Recently, the collisional and radiative atomic processes that couple energy levels have been shown to

* Corresponding author: hansen50@llnl.gov 
promote Boltzmann-type population distributions among levels within electronic configurations and among configurations within larger ensembles called superconfigurations [4]. The resultant effective temperature laws have been confirmed both analytically [2] and numerically [5-7], and suggest that some of the detailed structure of ions in plasma can be adequately described using statistical methods rather than explicit treatment $[8,9]$. To exploit this simplification of the general collisional-radiative (CR) problem, various superconfiguration models which statistically average detailed level structure into large ensembles have been developed [10-12].

With the significant compression of level structure achieved through the SC approach, non-LTE plasma ions can be described with a completeness that is computationally prohibitive for more detailed models $[2,13,14]$. The advantage of this compression can hardly be overstated: for many-electron systems, the number of fine structure levels can easily reach several million, pushing the limits of practical computing capabilities. Averaging these levels into a few hundred SC ensembles makes complex systems eminently tractable. However, in order for the computational advantages of the SC approach to be fully utilized, it must be proven to be reliable. Recently, successful comparisons of the results of SC models with experiments have been made $[2,6,11,14,15]$, and iterative models which split SCs into successively smaller ensembles have been shown to converge [13], suggesting internal consistency between detailed-configuration accounting (DCA) and SC models. But while the temperature law foundations of the SC approach have been numerically confirmed by detailed-level accounting (DLA) models (sometimes also called detailed term accounting, or DTA) $[5,6]$, to our knowledge no direct and systematic comparison between the results of SC and DLA models has yet been made.

In this work, we present a direct comparison between two independent models of neon: the SC model MOST [12] and a detailed-level accounting model. The low-Z test system was chosen so that a DLA model of reasonable size and sufficient completeness could be constructed for comparison with the SC model over a wide range of plasma conditions. While the relatively small modeled system accommodates the practical computational limits of the DLA approach, it challenges the statistical foundations of the SC approach: A few SCs in the present system contain only a single level, and the configurations and superconfigurations of the modeled K- and L-shell ions are generally much smaller than those of the M-shell systems treated by MOST in previous work $[6,12]$. Nonetheless, MOST gives good agreement with the DLA model for average ion charges and ion populations across a wide range of temperatures and densities and predicts reasonable values for effective temperatures within ions and SCs.

In the following sections, we: 
(1) describe the SC and DLA approaches and the modeled neon system,

(2) compare SC and DLA results for average ion charge, SC populations, and various effective temperatures, and

(3) develop an analytical formulation of a general effective temperature as a heuristic tool.

\section{Description of Models}

Two collisional-radiative models have been constructed to describe the nonLTE populations of neon ions in plasma. Both models are based on the same relatively simple and reasonably complete set of energy levels in neon, but while the DLA model treats each of more than 4500 fine-structure levels separately, the SC model groups those same levels into fewer than 50 large superconfigurations. (It is worth noting that even this hundredfold compression is much less than the compression that can be achieved for more complex systems.)

In the DLA model, the energy level structure and atomic rate data were generated by the original version of the HULLAC suite of atomic codes [1619], which are fully relativistic and include configuration-interaction effects. All levels within configurations formed by a promotion of the valence electron to $n$ le 4 are included in $\mathrm{H}$-like to $\mathrm{F}$-like neon, as are levels in configurations formed by promotion of a $1 s$ electron to $n$ le 3 in He- to F-like ions. These levels are coupled by eight atomic processes: spontaneous radiative and Auger decay, collisional excitation and de-excitation, collisional ionization and three-body recombination, dielectronic capture, and radiative recombination. Steady-state level populations are determined by solving the set of coupled linear equations (one for each level) formed by balancing the rates of population entering and leaving each level.

The energy level structure of the SC model MOST consists of pairs of Layzer's complexes, which ensemble-average configurations into non-relativistic superconfigurations according to the occupation numbers of the $n$ shells. Configuration energies and rates between configurations are calculated by the AVERROES suite of codes [14], which include relativistic corrections but not configuration interaction. MOST uses correlation properties of the AVERROES DCA system to obtain two sets of linear equations: one which gives the populations of the superconfigurations and another which give effective temperature describing the population of configurations within each SC [12].

Figure 1 shows the energy level structure of neon ions from the bare nucleus to the F-like ion, with each state represented by a horizontal line plotted with its energy relative to the ground state of neutral neon. The SC notation given 

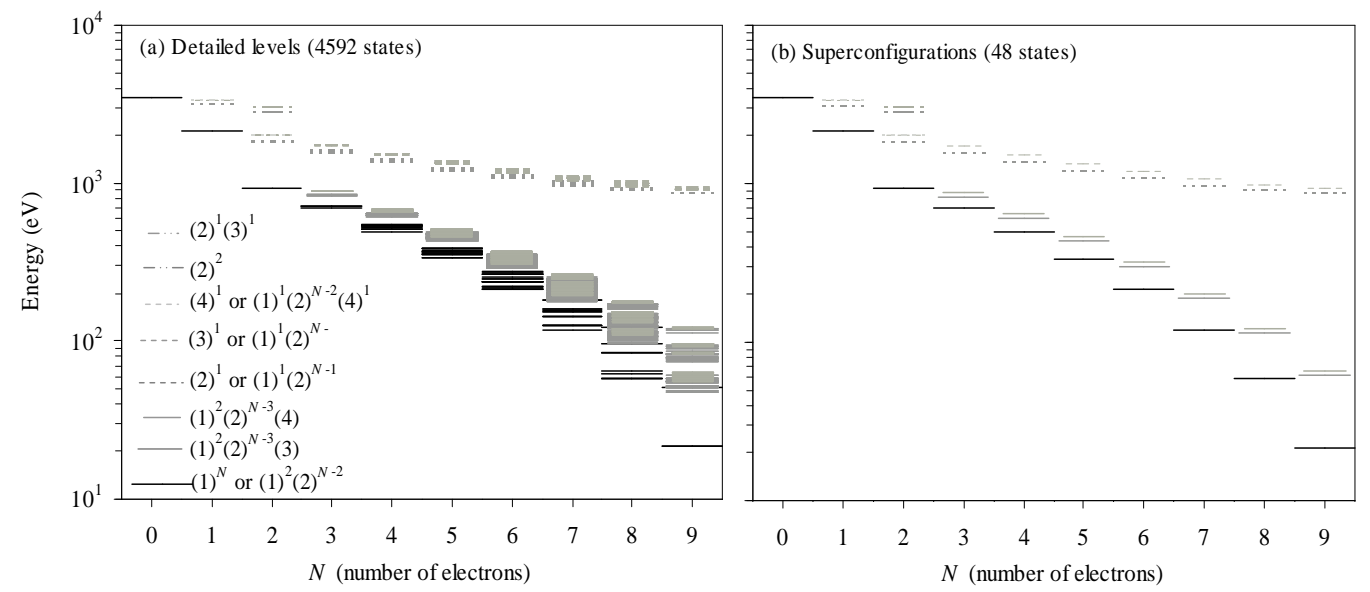

Fig. 1. Energies of states in (a) the DLA model and (b) MOST relative to the ground configurations of neutral neon.

in Fig. 1 (a) is based on the occupation number of the $n$ shells; for example, $(1)^{2}(2)^{3}$ comprises all configurations with 2 electrons in $n=1$ and 3 electrons in $n=2$ (i.e. $1 s^{2} 2 s^{2} 2 p, 1 s^{2} 2 s 2 p^{2}$, and $\left.1 s^{2} 2 p^{3}\right)$. Superconfiguration energies are calculated using

$$
E_{S C}=\sum_{i}\left(g_{i} E_{i}\right) / \sum_{i} g_{i}
$$

where $i$ runs over all configurations within the SC in MOST and all finestructure levels within the SC in the DLA model. (This construction and others like it in this paper are purely geometrical and do not include Boltzmann factors, which may be important in other contexts.) The $E_{S C}$ extracted from the HULLAC-based DLA model agree with the energies used in MOST to within a few percent, with the worst agreement $(\approx 2 \%)$ for highly excited levels in the near-neutral ions.

\section{Comparison of SC and DLA model results}

\subsection{Charge state distributions}

We have performed comparisons of the SC and DLA models over a wide range of plasma conditions at various levels of detail. The grossest such comparison is of the average ion charge $\langle Z\rangle$, which is shown in Fig. 2 for three electron temperatures $T_{e}(25,50$, and $100 \mathrm{eV})$ for electron densities $n_{e}$ from $10^{15}$ to $10^{22} \mathrm{~cm}^{-3}$. The solid black and short-dashed gray lines give the results of the DLA and SC models, respectively, and show good agreement between the 


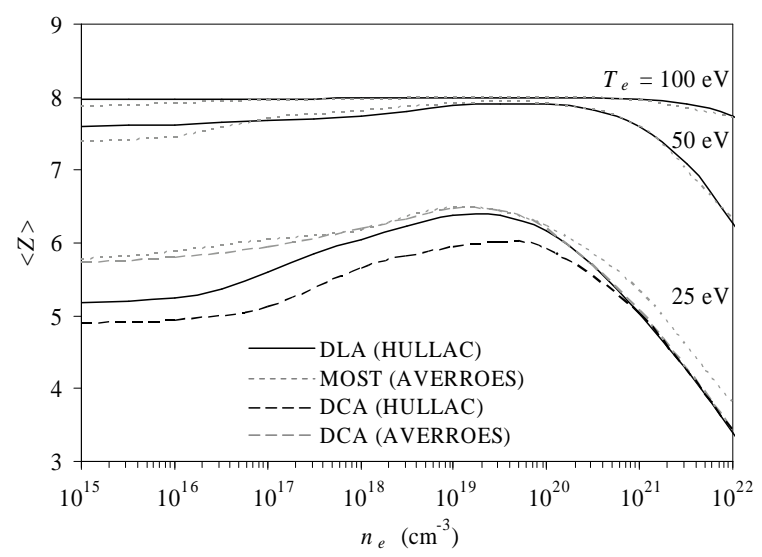

Fig. 2. Average ion charge from the DLA model and MOST as a function of $n_{e}$ for three values of $T_{e}$. For the $25 \mathrm{eV}$ case, results from DCA models based on HULLAC and AVERROES data are also given, showing that the primary source of disagreement arises from input data.

two models at high $T_{e}$ (where population collects in the He-like ion) and high $n_{e}$ (where the system approaches LTE). Both models predict an increase in $\langle Z\rangle$ at moderate electron densities as two-step (ladder) ionization becomes important, and then predict decreasing $\langle Z\rangle$ on the approach to LTE as threebody recombination becomes dominant.

There are significant discrepancies between the two models at low temperatures and densities: in the low-density limit of the $25 \mathrm{eV}$ case, the DLA model predicts a $\langle Z\rangle$ that is $10 \%$ lower than that predicted by MOST. This disagreement could be caused either by the averaging intrinsic to MOST or by differences in the input data. To distinguish between these two possibilities, we constructed detailed configuration-average (DCA) models from both the HULLAC and AVERROES data sources: The DCA results are given in Fig. 2 by long-dashed black and gray lines for the HULLAC and AVERROES data sets, respectively. The good agreement between the DCA model based on AVERROES data and MOST indicates that the SC-averaging procedure does not introduce intrinsic error, and points to the independent data sources as the cause of the disagreement. Part of this disagreement is attributable to additional dielectronic recombination channels in the DLA model due to configuration interaction between configurations in the singly excited $(1)^{2}(2)^{N-3}(3)$ and $(1)^{2}(2)^{N-3}(4)$ SCs in B- to F-like neon (see Fig. 1).

A final note of interest from Fig. 2 is the lingering disagreement between the DCA and DLA models based on HULLAC data. The DLA model is more highly ionized than the DCA model due to anomalously high populations of metastable excited levels in ground configurations, which can contribute to ladder ionization in the DLA model even at low densities. A hybrid model 
which retains fine-structure detail in the ground configurations of the ions matches the DLA results at low densities. Thus, we observe that while very little accuracy is lost in the transition from DCA to SC models (supporting the convergence suggested in [13]), there is some sacrifice of accuracy in moving from DLA to DCA models.

\subsection{SC populations and effective temperatures within ions}

Moving to an even finer level of detail, Fig. 3 shows normalized SC populations of He- through B-like neon at two electron densities with $T_{e}=50 \mathrm{eV}$. The normalized SC populations are the SC densities divided by the total SC statistical weights; they are given directly by MOST and are calculated in the DLA model using

$$
X_{S C} / g_{S C}=\sum_{i} X_{i} / \sum_{i} g_{i}
$$

where $X_{i}$ is the population density (in $\mathrm{cm}^{-3}$ ) of states in the level $i$ and the sum runs over all levels in each SC. The solid and dashed lines in Fig. 3 give the best-fit lines to a Boltzmann-type distribution of SC populations for each ion; their slopes represent an effective ion temperature $T_{i o n}$ which describes the populations of the superconfigurations through $X_{S C} / g_{S C} \propto e^{-E_{S C} / T_{i o n}}$.

In the high-density case [Fig. 3 (a)], the DLA model and MOST are in excellent agreement for both the SC populations and the derived ion temperatures. The agreement remains fairly good in the lower-density case [Fig. 3 (b)] for all ions except He-like neon, where the disagreement is likely due to the small number of levels in the He-like SCs. Since the SC model only promises to be reliable when statistical correlations between large ensembles hold, the disagreement here is not surprising. Indeed, we find generally that the quality of the agreement in the SC populations is directly correlated with the degeneracy of the SCs.

We note in particular that while the DLA model predicts lower populations than the SC model for most of the He-like neon SC populations in Fig. 3 (b), the DLA model predicts a higher population for $(1)^{1}(2)^{1}\left(E_{S C}=914 \mathrm{eV}\right)$. This is due to the presence of a metastable level in the $1 s 2 s$ configuration which has no strong decay channel to $1 s^{2}$ and so collects population through radiative cascades from other excited levels. In the SC model, the metastable behavior is washed out by averaging with the strong $1 s 2 p-1 s^{2}$ decay channel. This averaging has two consequences for any pure SC model: First, since ionization balances can be driven by ladder ionization from metastable states with significant population even at low densities, SC models may tend to slightly 

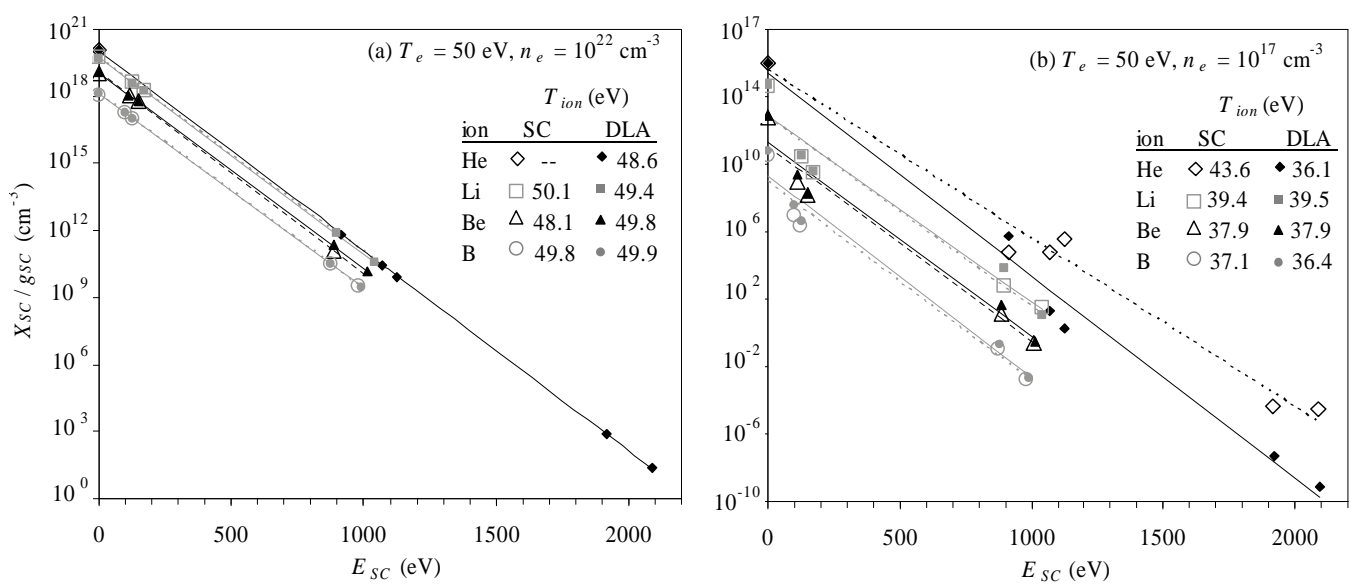

Fig. 3. Normalized SC population densities at $T_{e}=50 \mathrm{eV}$ and $n_{e}=$ (a) $10^{22}$ and (b) $10^{17} \mathrm{~cm}^{-3}$ given by closed and open points for the DLA model and MOST, respectively. Ion temperatures describing the distribution of SC populations with $\mathrm{SC}$ energies are listed on the plots and represented by solid and dashed lines for the DLA model and MOST, respectively. and are also .

underpredict $\langle Z\rangle$ (A similar effect was observed in the HULLAC-based DCA model discussed above.) Second, widely-used density diagnostics based on line emission from closed-shell ions whose low-lying states collect population at low electron densities will not be accessible when the metastable states are ensemble-averaged. These caveats are in any case beyond the claims made by the SC approach, and, since they tend to be most important for closedshell ions, can be corrected with modest concessions to complexity by using restricted DLA or hybrid models.

In the high-density case given in Fig.3 (a), the SC populations lie very close to the straight lines describing the Boltzmann distributions, and the derived $T_{i o n}$ are very close to $T_{e}$, reflecting the proximity of the system to LTE. In the lower-density case where the system is far from LTE, the derived $T_{i o n}$ are significantly smaller than $T_{e}$, and the populations of levels with $E_{S C} \approx$ $200 \mathrm{eV}$ deviate significantly from the Boltzmann distributions. If the highenergy SCs formed by excitation of a $1 s$ electron in the L-shell ions are neglected, the derived $T_{i o n}$ falls from $\approx 40 \mathrm{eV}$ to $\approx 10-15 \mathrm{eV}$. The resulting Boltzmann distributions describe the populations of the lower-energy SCs very well, but significantly underpredict the populations of the high-energy SCs. We have found that the high-energy SCs in the L-shell ions are of very little importance to the ionization dynamics and might as well have been neglected altogether in the present model. However, their anomalous behavior at low densities indicates that the notion of a universal effective temperature which is independent of level energy is problematic, and underscores the importance of including state-dependent collisional-radiative effects in the calculation of effective temperatures (as is done explicitly by MOST through correlations 

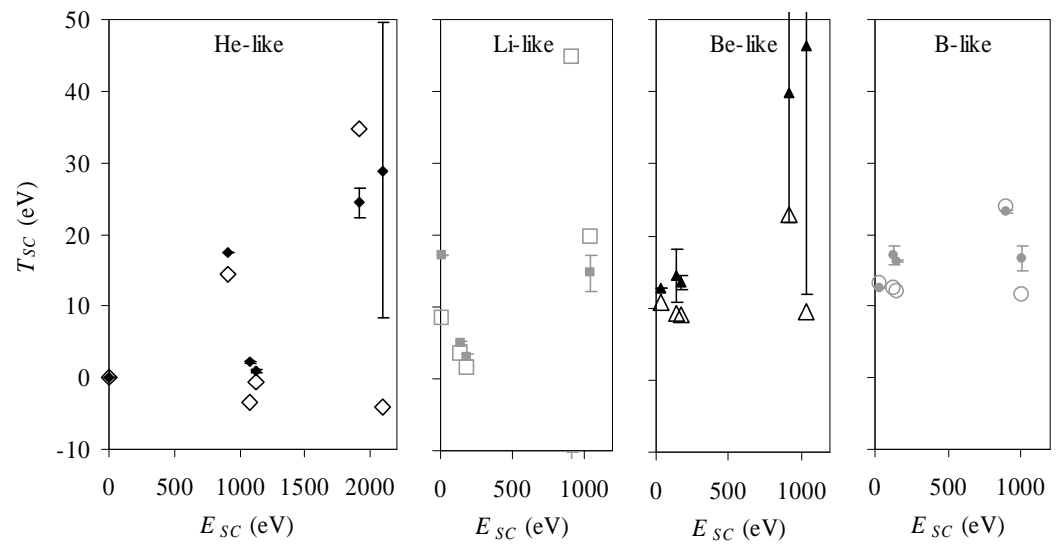

Fig. 4. Superconfiguration temperatures describing the distribution of configuration populations within SCs at $T_{e}=50 \mathrm{eV}$ and $n_{e}=10^{17} \mathrm{~cm}^{-3}$. Closed and open points give $T_{S C}$ from the DLA model and MOST, respectively. Error bars derived from correlation coefficients are given for the DLA points.

and implicitly in the DLA model by averaging over CR level populations).

\subsection{Effective temperatures within SCs}

The effective ion temperature defined in the previous section described the distribution of SC populations in each ion. Another effective temperature generated by both the DLA model and MOST is the superconfiguration temperature $T_{S C}$, which describes the distribution of configurations within each superconfiguration. In MOST, $T_{S C}$ are calculated directly from a system of linear equations generated through correlations of rates coupling configurations. In the DLA model, $T_{S C}$ are determined by finding the best-fit line of the natural logarithm of normalized configuration populations $X_{C} / g_{C}$ to the configuration energies $E_{C}$. Explicitly, $E_{C}$ and $X_{C} / g_{C}$ are calculated in the DLA model by

$$
\begin{aligned}
& E_{C}=\sum_{i}\left(g_{i} E_{i}\right) / \sum_{i} g_{i} \\
& X_{C} / g_{C}=\sum_{i}\left(X_{i}\right) / \sum_{i} g_{i}
\end{aligned}
$$

In Eqs. (3) and (4), the summations run over all levels within each electronic configuration.

Figure 4 gives a comparison of the superconfiguration temperatures obtained with the DLA model and MOST for $T_{e}=50 \mathrm{eV}$ and $n_{e}=10^{17} \mathrm{~cm}^{-3}$. The 

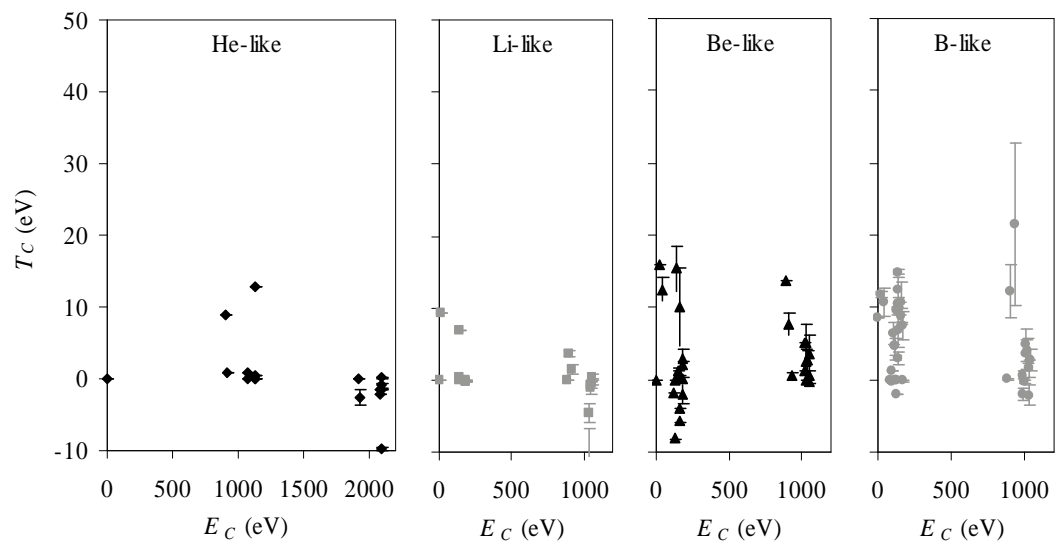

Fig. 5. Configuration temperatures describing the distribution of levels within configurations at $T_{e}=50 \mathrm{eV}$ and $n_{e}=10^{17} \mathrm{~cm}^{-3}$ in the DLA model.

magnitudes of the error bars given for the DLA data points are derived from the correlation coefficient $r$ of the linear least-squares fit through $T_{S C}(1-r)$. The agreement between MOST and the DLA models in $T_{S C}$ is quite good for the low-energy SCs formed by excitation of a valence electron. The higherenergy SCs have larger disagreements in $T_{S C}$ but tend also to have larger error bars. The typical values for $T_{S C}$ of $10-20 \mathrm{eV}$ are smaller than the $\approx 40 \mathrm{eV}$ ion temperatures derived using the full set of SCs at the same plasma conditions, and are on the whole less predictable than $T_{i o n}$, which varied little between ions.

\subsection{Effective temperatures within configurations}

Yet another effective temperature, the configuration temperature $T_{C}$, can be defined to describe the population distribution of levels within configurations. The SC model offers no predictions for $T_{C}$, but we include here the predictions of the DLA model for completeness. Figure 5 gives configuration temperatures from the DLA model at $T_{e}=50 \mathrm{eV}$ and $n_{e}=10^{17} \mathrm{~cm}^{-3}$ for He- through B-like neon ions. The configuration temperatures generally fall between -10 and 10 $\mathrm{eV}$ (negative effective temperatures signal population inversions which occur between metastable levels and levels with large radiative decay rates).

Since collisional processes enforce LTE most readily among levels with small energy separations, it would be reasonable to expect effective temperatures to increase towards $T_{e}$ as the ensemble size (and thus the energy separation) decreases. However, the three sets of effective temperatures so far presented exhibit a somewhat surprising trend of decreasing effective temperatures with decreasing ensemble size. On the side of the smallest ensembles considered 
(levels within configurations), the deviation from our expectation is largely due to strong radiative decay channels which couple excited levels more strongly to ground states than to nearby excited levels at even moderate densities. On the side of the large ensembles (superconfigurations within ions), we have already described how anomalously large populations of high-energy SCs force the effective temperature towards $T_{e}$. A heuristic tool for understanding this behavior is given in the next Section.

\section{Analytical formulation of effective temperature}

To aid our understanding of the collisional-radiative foundations of population distributions, we can derive an expression for an effective temperature $T_{\text {eff }}$ in a simple two-level system. Consider an excited level $\left(X_{1}\right)$ which lies $\Delta E$ in energy above the ground level $\left(X_{0}\right)$. In equilibrium, the level populations $X$ and the collisional-radiative rates $R$ coupling the two levels are balanced: $X_{0}$ $R(0 \rightarrow 1)=X_{1} R(0 \rightarrow 1)$. Let $T_{\text {eff }}$ be the temperature which enforces the collisional-radiative population balance through a Boltzmann relation

$$
\frac{X_{1}}{X_{0}}=\frac{R(0 \rightarrow 1)}{R(1 \rightarrow 0)}=\frac{g_{1}}{g_{0}} e^{-\Delta E / T_{e f f}}
$$

If the two levels belong to the same ion and are coupled by a dipole radiative decay rate, then $R(0 \rightarrow 1)$ contains only a collisional excitation rate and $R(1 \rightarrow 0)$ contains collisional de-excitation and spontaneous radiative decay rates. With the Van Regemorter approximation [20] for the collision strength, all three of these rates are proportional to the dipole oscillator strength and their ratio is independent of the details of the dipole coupling. Using the Gaunt factors recommended by Mewe [21] in the collisional rates, the effective temperature is implicitly given by

$$
e^{-\Delta E / T_{e f f}}=e^{-\beta}\left[1+\frac{2.75 \times 10^{12}}{0.15+0.28 \chi(\beta)}\left(\frac{\Delta E^{3} T_{e}^{1 / 2}}{n_{e}}\right)\right]^{-1}
$$

where $\beta=\Delta E / T_{e}$ and $\chi(\beta)=\ln \left[1+(0.562+1.4 \beta) /\left(\beta+1.4 \beta^{2}\right)\right][22]$ with $n_{e}$ in $\mathrm{cm}^{-3}$ and $T_{e}, T_{e f f}$, and $\Delta E$ in $\mathrm{eV}$. The term containing $\Delta E^{3} T_{e}^{1 / 2} / n_{e}$ represents the branching ratio of radiative decay to collisional de-excitation. Note that while Eq. 6 is valid only for optically thin plasmas with Maxwellian electron distributions, Eq. 5 is general and can be modified to include collisional rates driven by non-Maxwellian distributions and stimulated emission and absorption rates driven by opacity effects or external radiation fields. 

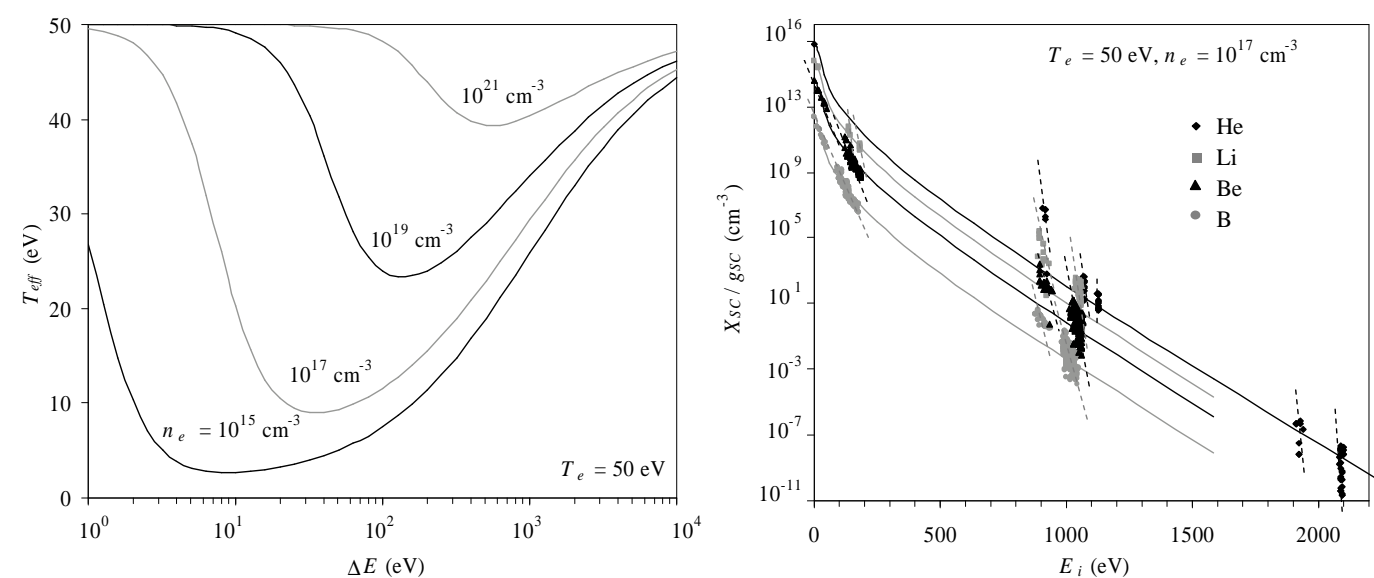

Fig. 6. Left panel: Analytical $T_{e f f}$ vs. $\Delta E$ at $T_{e}=50 \mathrm{eV}$ and various $n_{e}$. Right panel: Normalized DLA level populations at $T_{e}=50 \mathrm{eV}$ and $n_{e}=10^{17} \mathrm{~cm}^{-3}$. Solid lines are Boltzmann distributions at $T_{e f f}\left(E_{i}\right)$ and dashed lines are Boltzmann distributions within $\mathrm{SC}$ ensembles with characteristic energy separations $\Delta E_{S C}$ and $T_{\text {eff }}\left(\Delta E_{S C}\right)$.

The left panel of Fig. 6 illustrates the dependence of the effective temperature given by Eq. 6 on the electron density and the energy separation of the two levels. For any $\Delta E$, a sufficiently large $n_{e}$ can ensure the dominance of collisional processes, moving the branching ratio towards zero and enforcing LTE through detailed balance. And at moderate densities, decreasing energy separations lead to small radiative decay rates, which also decrease the branching ratio and encourage LTE. Both of these effects are expected. But when the energy separation is large $\left(\Delta E \gg T_{e}\right)$, the effective temperature increases with $\Delta E$, an effect mirroring the surprisingly high populations of the high-energy SCs observed in both the DLA model and MOST.

The right panel of Fig. 6 gives the normalized level populations of the DLA model at $T_{e}=50 \mathrm{eV}$ and $n_{e}=10^{17} \mathrm{~cm}^{-3}$. Boltzmann distributions obtained using the analytical effective temperature with $\Delta E=E_{i}$ are given by solid lines and reproduce the global populations of level clusters within ions rather well. The analytical effective temperature also does a fair job of estimating the population distributions of levels within superconfigurations (given by dashed lines) when $\Delta E$ is taken to be the characteristic energy separation of levels within the SCs. We note that the present analytical formulation accounts for only three atomic processes, all within a single ion. Addressing the population balance between ions may require consideration of further processes. 


\section{Conclusions}

We have compared average charge states, charge state distributions, superconfiguration populations, and effective ion and SC temperatures from a DLA model and the SC model MOST across a wide range of plasma conditions for a relatively small but complete test system of neon ions. Aside from some disagreement for ensembles comprising a small number of levels, the SC model predictions agree quite well with the DLA model results for SC populations and effective temperatures. Considering the inherent difficulties for the SC approach in the selected model system, the present comparisons can reasonably support high hopes for the reliability of the SC approach, particularly for more complex systems with larger SC ensembles. In addition, we have derived a simple analytic formula for a general collisional-radiative effective temperature which heuristically describes the behavior of effective temperatures within various level ensembles.

\section{Acknowledgements}

This work was performed under the auspices of the U.S. Department of Energy by University of California Lawrence Livermore National Laboratory under contract No. W-7405-Eng-48.

\section{References}

[1] Busquet M. Radiation-dependent ionization model for laser-created plasmas. Phys Fluids B 1993;5:4191-4206.

[2] Bauche J, Bauche-Arnoult C. Temperatures in non-LTE hot plasmas. J Phys B 2000;.33:L283-L288.

[3] Blancard C, Faussurier G, Kato T, More RM. Effective Boltzmann law and Prigogine theorem of minumun entropy production in highly-charged ion plasmas. J Quant Spectrosc Radiat Transf 2004 (this volume).

[4] Bauche-Arnoult C, Bauche J. The temperature law for superconfigurations in non-LTE plasmas. J Quant Spectrosc Radiat Transfer 2001;71:189-199.

[5] Fournier KB, Bauche J, Bauche-Arnoult C. Evidence for a temperature law in non-LTE plasmas. J Phys B 2000;33:4891-4904.

[6] Bauche J, Bauche-Arnoult C, Peyrusse O, Bachelier A, Fournier KB, ChenaisPopovics C, Gauthier J-C. Analysis of a non-LTE xenon spectrum by means of the model of superconfiguration temperatures. J Quant Spectrosc Radiat Transfer 2003;81:47-55. 
[7] Busquet M. Onset of pseudo-thermal equilibrium within configurations and superconfigurations. J Quant Spectrosc Radiat Transfer 2004 (this volume).

[8] Bar-Shalom A, Oreg J, Goldstein WH, Shvarts D, Zigler A. Super-transitionarrays: a model for the spectral analysis of hot, dense plasmas. Phys Rev A 1989;40:3183-3193.

[9] Bar-Shalom A, Oreg J, Goldstein WH. Effect of configurations widths on the spectra of local thermodynamic equilibrium plasmas. Phys Rev E 1995;51:4882-4890.

[10] Bar-Shalom A, Oreg J, Klapisch M. Recent developments in the SCROLL model. J Quant Spectrosc Radiat Transfer 2000;65:43-55.

[11] Faussurier G, Blancard C, Berthier E. Nonlocal thermodynamic equilibrium self-consistent average-atom model for plasma physics. Phys Rev E 2001;63:026401-1-13.

[12] Bauche J, Bauche-Arnoult C, Fournier KB. Model for computing superconfiguration temperatures in nonlocal-thermodynamic-equilibrium hot plasmas. Phys Rev E 2004; x:026403-1-10.

[13] Bar-Shalom A, Oreg J, Klapisch M. Collisional radiative model for heavy atoms in hot non-local-thermodynamical-equilibrium plasmas. Phys Rev E 1997;56:R70-R73.

[14] Peyrusse O. A superconfiguration model for broadband spectroscopy of nonLTE plasmas. J Phys B 2000;33:4303-4321.

[15] Peyrusse O. On the superconfiguration approach to model NLTE plasma emission. J Quant Spectrosc Radiat Transfer 2001;71:571-579.

[16] Klapisch M, Schwob J, Fraenkel B, Oreg J. The 1s-3p Kbeta-like X-ray spectrum of highly ionized iron. J Opt Soc Am 1977;67:148-155.

[17] Bar-Shalom A, Klapisch M, Oreg J. Electron collision excitations in complex spectra of ionized heavy atoms. Phys Rev A 1988;38:1773-1784.

[18] Bar-Shalom A, Klapisch M. NJGRAF - An efficient program for calculation of general recoupling coefficients by graphical analysis, compatible with NJSYM. Computer Phys Comm 1988;50:375-393.

[19] Oreg J, Goldstein WH, Klapisch M, Bar-Shalom A. Autoionization and radiationless electron capture in complex spectra. Phys Rev A 1991;44:17501758.

[20] Van Regemorter H. Rate of Collisional Excitation in Stellar Atmospheres. Astrophys J 1962;136:906-915.

[21] Mewe R. Interpolation formulae for the electron impact excitation of ions in the H-, He-, Li- and Ne-sequences. Astron Astrophysics 1972;20:215-221.

[22] Shevelko VP. Atoms and Their Spectroscopic Properties Berlin:SpringerVerlag, 1997. p. 149. 